\title{
Research on the Current Situation of Traditional Martial Arts Inheritance
}

\author{
Ruimin Ji \\ Martial Arts Department of Chengdu Sports University, Chengdu, China
}

Keywords: Traditional martial arts, Protection mechanism, Inheritance, History, Dilemma

\begin{abstract}
This document explains and demonstrates how to develop the traditional martial arts inheritance protection mechanism paradigm. Through the research on the real situation of traditional martial arts inheritance people and an analysis of the current situation of the development of traditional martial arts, the inheritance person protection mechanism are introduced in the importance of the development of traditional martial arts.Searching for the future road of the protection of traditional culture development and providing theoretical reference and practical reference can enrich the practice of China's cultural soft power resources construction.
\end{abstract}

\section{Introduction}

Inheritance is the information carrier and the inheritance entity of, and inheriting people focus on boxing foremost. All kinds projects of refer to the orderly, clear, unique boxing and cold weapon technology formatting since Ming and Qing dynasty. Every kind of boxing has its unique style, technique, practice program and suitable crowd with the complete technical training system.

\section{Interpretation of the traditional martial arts inheritance protection mechanism paradigm}

Theory of traditional wushu inheritance. Historically boxing was the main continuation of traditional martial arts which was carried by "living heritage" carrier who would use oral and body as the basic method for generations continue down. Inheriting people is the core carrier of mastering and carrying the traditional martial arts knowledge and consummate skill, playing the key role in the inheriting of boxing. The determination of the status of the boxing inheritors mainly depends on the public's recognition of the identity of the inheritors.

By inheriting the practice of past legacy completely and systematically, the inheriting people are usually in the hands of the technology about the door shrouded in secrecy system and cultural system. Once accident happened, a kind of boxing may lead to the fault.According to the essence of the intangible cultural heritage inheritance people attribute and feature, this study discusses that the traditional martial arts inheritance person refers to one who inherit a king of boxing completely and systematically and is highly known as a skilled descendant.

The traditional martial arts protection mechanism combing. Zhang shixian believes that protection is using all possible means to preserve the status of human cultural assets in the past and prolong the life of the living. Xie Ninggao proposed that the authenticity of heritage protection is to keep the meaning of heritage, integrity and diversity, and that its value from sudden threat or a progressive damage factors, enable it to inscribe it sustainable development and the sustainable utilization. Above all scholars understanding of "protection" , to protect the definition essentially still exist in common protection is for the purpose of prolonging the life of the heritage, in order to provide use for the future.

In the field of cultural heritage, "protection" is the core and root of the heritage value system and the object system, and is also one of the characteristics of heritage research. For all cultural heritages, the primary meaning of "protection" is "preservation", which can be used to preserve the existing material form effectively and make it permanent. However, intangible cultural heritage which depends on individuals, groups, specific regions or spaces and is a living culture differs from material 
cultural heritage. "Protection", therefore, is more than a physical form "save" which needs to collect and preserve heritage material carrier of the living condition culture, through various means to make them physical form and ensure continuation in the modern society .According to "Convention on the Protection of Intangible Cultural Heritage", protection is defined as taking measures to ensure the vitality of intangible cultural heritage, including study, preserve, protect, propaganda and other aspects of the heritage recognition. The study considers that the protection mechanism is a systematic and sustainable operation mode which aims at prolonging the life of intangible cultural heritage. The protection mechanism of traditional martial arts is a stable and sustainable operation mode which aims at protecting the inheritors' training of the successors.

\section{The realistic demand of traditional martial arts development}

The cultural origin of traditional martial arts. Chinese martial arts grew as early as the pre-qin period, from simple fighting skills of catching food and animal to the organized instruments and unarmed fighting among tribe wars.

The frequent wars were a great hindrance to the survival and development of the tribes, and in order to show the war capabilities of the tribes, the primitive people created a martial dance, called war dance. At the same time, the war also led to the emergence of weapons. The archery in the stone age and the bronze spear and sword in shang and zhou dynasties marked the beginning of age of Chinese martial arts culture.

During the period of the spring and autumn period and the warring states period, labor productivity rose greatly and the economy boomed, but it did not avoid frequent wars, which has given rise to the social warrior. Then the martial arts system has been formed, and the martial arts function has been gradually diversified, which has been highlighted as the performance, competition and entertainment. Meanwhile, the pre-qin scholars endowed Chinese martial arts with rich and colorful cultural connotation, not only laying the important historical conditions, ,but becoming the constant thought source for the development of traditional martial arts for over two thousand years. Influenced by the culture during this period, the traditional martial arts developed in a harmonious, perfect, ideal human environment with characteristics of culture of the unity of inside and outside. Follow the unity of human and nature, follow the objective law, and maintain a high harmony and unity with it.

Secondly, the Confucian principles of moral law also laid down the rules of practice. The thought of eastern Confucianism occupies the dominant position in the feudal society for more than two thousand years. Under its influence, the martial arts circle has formed the common standard and standard of wide.

Thirdly, the special category of oriental classical aesthetics infiltrates traditional martial arts.

In Chinese ancient aesthetic category, there are aesthetic artistic conceptions of qi and rhyme, shape, spirit, meaning, masculine and feminine. The traditional martial arts not only requires external action with physical beauty, but also pays attention to the lofty stature, making a harmony of thought and action. Boxing starts from Yi, theory is influenced by medicine. Integrating traditional medical theories into martial arts greatly enriches the traditional martial arts theory system, which makes it differ from other sports items. Both martial arts and military science reflect a typical oriental cultural consciousness and cultural psychological structure. So Chinese martial arts are the congealing and presenting of the eastern military culture.

For a long time in history, martial arts were closely related to the military. As a means to overcome the enemy, martial arts has demonstrated the strategic thinking of ancient military law. Traditional martial arts have its unique culture charisma, attracting some foreigners coming to China to study martial arts. Actually they are not only obsessed with a kind of boxing or surface technology, but hope understand Chinese splendid civilization, which is the charm of traditional martial arts.

The survival situation of traditional martial arts succession. At present, the problem of intangible cultural heritage is lack of inheritors and the participation of a new generation of young talents. Traditional martial arts were no exception, facing the problem of lack of inheritors. In the process of catering to the times, traditional martial arts have become a variety of forms of competitive martial arts, traditional martial arts and popular martial arts, but its core is gradually forgotten and 
annihilated. Although the traditional portal concept of martial arts gradually has been broken and multiple practiser opened free learning opportunities, increasing the number of people involved in practicing to a certain extent, only a few young people and children participated. Moreover, there are still many traditional martial arts students inheriting the traditional insemination mode, which limits their wide spread.

Therefore, the main common inheritance difficulty of traditional martial arts is the absence of inheritors, especially the lack of the participation of more young talents. The lack of the inheritance of young people and the gradual aging of the old generation are the first key difficulties in the inheritance.

\section{Summary}

After analyzing and summarizing, it is believed that the protection mechanism of traditional martial arts is not yet perfect, and there are several reasons for the lack of traditional martial arts culture.

Guidance of aesthetic tendency. As a traditional culture, aesthetic value of martial arts is contradictory to the aesthetic taste of young people advocating modern and modern culture. For example, teenagers are willing to learn taekwondo, rather than practice traditional martial arts. Therefore, how to make the traditional martial arts modern , charm and more attractive to the youth, it is urgent to innovate authorization method as well as the teaching content.

The shackles of the concept of inheritance. Since ancient times, many martial arts scholars have kept such a family, which has continued to this day and became the cultural tradition of Chinese martial arts inheritance. However, in the rapid development of cultural globalization, traditional martial arts has gradually lost its charm, and traditional boxing concept has further removed from the daily life of the masses. Therefore, breaking down the shackles of the concept of inheritance and making the traditional martial arts re-enter people's daily life might be able to save the traditional martial arts that are being annihilated.

Arbitrariness of the boxing mode. The mode of boxing of folk traditional martial arts is basically "the teaching of the master, the diligence of the apprentice". Traditional martial arts not appeal to teens who are relative to the nature fun, activities and active, which greatly influenced the participate in the practice of traditional martial arts practitioner population. Therefore, how to attract more young people from traditional martial arts is the key to the traditional martial arts.

\section{Acknowledgements}

This research was financially supported by the National Martial Arts Management Center project (youth project) -- Research on Protection Mechanism of Traditional Martial Arts Inheritors.

\section{References}

[1] Wang Jianjun. The impact of the new rules of wushu on the creation of competition routines [J]. Sports world (academic edition), 2015(5).

[2] Yang Yue. A little discussion on the protection mechanism of the inheritance of intangible cultural heritage in China. [J] ,Caizhi,2013,09(05)

[3] li ning. Research on sustainable development of Chinese traditional wushu [D]. Shandong normal university, 2009.

[4] Wang Gang. Chinese martial arts: a demonstration of the culture of military culture [J]. Journal of tianjin sports institute, 2007,11(10). 